STUDI

FRANCESI

\section{Studi Francesi}

Rivista quadrimestrale fondata da Franco Simone

190 (LXIV | I) | 2020

Varia - fasc. I - gennaio-aprile 2020

\title{
FRANÇOIS AMY DE LA BRÉTÈQUE, L'Imaginaire médiéval dans le cinéma occidental
}

\author{
Maria Colombo Timelli
}

\section{OpenEdition}

\section{Journals}

\section{Édition électronique}

URL : https://journals.openedition.org/studifrancesi/22162

DOI : $10.4000 /$ studifrancesi.22162

ISSN : 2427-5856

\section{Éditeur}

Rosenberg \& Sellier

\section{Édition imprimée}

Date de publication : 1 avril 2020

Pagination : 141-143

ISSN : 0039-2944

\section{Référence électronique}

Maria Colombo Timelli, « FRAnçoIS AMY de LA BRÉTĖQue, L'Imaginaire médiéval dans le cinéma occidental 》, Studi Francesi [En ligne], 190 (LXIV | I) | 2020, mis en ligne le 01 avril 2020, consulté le 02 août 2021. URL : http://journals.openedition.org/studifrancesi/22162 ; DOI : https://doi.org/10.4000/ studifrancesi.22162

Ce document a été généré automatiquement le 2 août 2021

\section{(c) (i) (9)}

Studi Francesi è distribuita con Licenza Creative Commons Attribuzione - Non commerciale - Non opere derivate 4.0 Internazionale. 


\title{
FRANÇOIS AMY DE LA BRÉTÈQUE, L'Imaginaire médiéval dans le cinéma occidental
}

\author{
Maria Colombo Timelli
}

\section{RÉFÉRENCE}

FRANÇOIS AMY DE LA BRÉTÈQUE, L'Imaginaire médiéval dans le cinéma occidental, Paris, Champion, 2018, deux volumes, 1276 pp.

1 Paru en 2004 et réimprimé par Champion en 2018, cet ouvrage fondamental n'avait jamais été signalé dans "Studi francesi"; et s'il nous semble mériter une notice quelque peu développée, c'est que la réflexion qu'il propose pour une filmographie qui s'arrête aux années 2000, tout en gardant son intérêt, pourra facilement être appliquée aux productions de ces quinze dernières années.

2 Plutôt que dans le cadre d'une histoire des mentalités, l'étude de F. Amy de la Bretèque se situe dans une histoire des représentations, en l'occurrence des représentations collectives, en se donnant pour objet un corpus étendu: les «films commerciaux de fiction» (p.19) produits en Europe et aux États-Unis pour lesquels le Moyen Âge Moyen Âge central, prolongé jusqu'aux XIV et $\mathrm{XV}^{\mathrm{e}}$ siècles - constitue l'hypotexte (on trouvera la liste des 290 titres retenus en annexe, pp. 1099-1225).

3 Une première partie (pp. 25-185) aborde quelques Questions générales; premièrement, la centralité dans les productions de l'énonciation historique (chapitre 1), incarnée dans quelques indices: langue, realia, scènes récurrentes (adoubement, banquet, tournoi). Ces procédés ne sont pas sans danger: la reconstitution du Moyen Âge s'avère de fait impossible et les risques de surcharge sont toujours présents au croisement entre discours historiographique et discours fictionnel (chapitre 2). De même, conscient ou pas, l'anachronisme est inéluctable, sous trois formes au moins: anachronisme, prochronisme, parachronisme (chapitre 3). Quelques modèles pertinents pour l'analyse 
des productions cinématographiques sont repérés dans des domaines éloignés du cinéma: réflexions de Panovski sur motifs et thèmes, puis de Bakhtine, Kristeva et Genette sur l'intertextualité (chapitre 4). Plusieurs formes externes au cinéma se sont imposées sur les fictions: roman historique du $\mathrm{XIX}^{\mathrm{e}}$ siècle, manuels scolaires, roman "gothique», mais aussi opéra, opérette et «musical» anglo-saxon, peinture historique et préraphaëlite, jusqu'à des formes classées comme «populaires» (Bibliothèque bleue, lanterne magique, image d'Épinal, bande dessinée, théâtre de marionnettes), sans négliger le modèle historiographique savant, dont l'importance n'est cependant pas à surestimer (chapitre 5). Le corpus considéré est nécessairement hétérogène, au point qu'on peut douter qu'il s'agisse d'un seul et même "genre»; quelques formes se reconnaissent néanmoins: «Epic Film», mélodrame, film de chevalerie, entre autres (chapitre 6). Un dernier chapitre porte sur les premiers exemples de films «moyenâgeux» produits en France: ceux de Georges Méliès au début du xxe siècle et production de Gaumont et Pathé dans les années 1907-1914 (chapitre 7).

4 La deuxième section (La scène mythique, pp. 193-510) propose une analyse fine et serrée des films dont le cadre narratif et fictionnel est tiré de créations médiévales. On commence par les vies de saints, manipulées par le cinéma, et par l'exemple remarquable de saint François d'Assise (chapitre 8), pour passer rapidement aux personnages ayant formé le noyau de trois cycles cinématographiques: Roland et saga carolingienne, Siegfried et les Nibelungen, Le Cid et la Reconquista hispanique; les héros fondateurs de trois nations européennes, éventuellement soumis à des lectures nationalistes, ont inspiré en particulier le cinéma muet et celui des années 1960-1970 (chapitre 9). Le roi Arthur et la matière arthurienne ont constitué un terrain de prédilection pour le cinéma américain, qui n'hésite ni à traiter ses sources (et leurs nombreux intermédiaires) avec «désinvolture» (p. 276), ni à les plier au contexte contemporain et à ses préoccupations (chapitre 10). De même, la Table Ronde a souvent été chargée d'un message idéologique très éloigné des références médiévales: devenue une création humaine à finalité politique, elle perd son rôle central, alors que l'accent se déplace vers le groupe des chevaliers et les tensions qui les traversent (chapitre 11). D'autres héros arthuriens s'affirment en tant qu'individus en devenant les protagonistes de fictions autonomes: Gauvain et Lancelot au premier chef (chapitre 12). Le Graal et sa quête, «sans doute l'héritage le plus fort que nous ait légué le Moyen Âge dans le domaine de l'imaginaire» (p.361), devenu un véritable mythe rattaché au personnage de Perceval, a fourni matière à de très nombreux films, dont l'inspiration se différencie profondément entre productions germanique, hollywoodienne et française (chapitre 13). Rarement protagoniste, Merlin est pourtant souvent présent dans les films, parfois sous les formes d'autres enchanteurs et magiciens (chapitre 14). Les amours médiévales ne pouvaient évidemment pas manquer: la fin'amors des troubadours, Paolo et Francesca de Dante, l'amour courtois, le Roman de la Rose, les images de la fontaine et du verger, ont tous nourri le cinéma occidental (chapitre 15); dans ce domaine, la fortune de Tristan et Iseut, surtout en France, est connue, avec des choix d'épisodes récurrents: le Morholt, le philtre, la forêt du Morois, la mort des amants (chapitre 16). Au contraire, Renard constitue une sorte de «rendez-vous manqué» (p. 501, chapitre 17).

5 Dans la troisième partie (Le décor de l'histoire, pp. 513-901), les sujets proprement historiques sont abordés. Les Croisades, notamment la première et la troisième, ont finalement peu intéressé le cinéma, avec quelques exceptions en Italie, en France, et 
surtout aux États-Unis; de fait, plutôt que devenir l'objet de «reconstitutions» historiques, elles se sont transformées en hypotextes de discours plus conceptuels (chapitre 18). Les rois Plantagenêt de la fin du XII siècle ont largement contribué à créer non seulement des films, mais des séries télévisées, centrées notamment sur Yvanhoé et sur Robin des Bois (chapitre 19), puis sur Richard Cœur de Lion (chapitre 20). Mais c'est le théâtre shakespearien qui a fourni au cinéma le matériau le plus riche et surtout le filtre à travers lequel une large part de l'histoire médiévale a été représentée (chapitre 20). L'imaginaire filmique s'est aussi nourri à des phénomènes en partie seulement historiques: sorcellerie, hérésies, horreurs diverses (tortures de l'Inquisition, bûchers, révoltes des victimes), représentations de l'Enfer et du Paradis (chapitre 22). Quelques "grandes peurs» médiévales ont également inspiré les cinéastes: épidémies et peste noire, Danse macabre et Jugement Dernier (chapitre 23). Quant à la Guerre de Cent Ans, elle a souvent été interprétée, en France tout spécialement, comme un moment capital dans l'affirmation de la conscience nationale; si quelques figures et moments-clés émergent (Bertrand Du Guesclin, bataille d'Azincourt), ce conflit interminable s'est aussi prêté à une réflexion générale sur la guerre (chapitre 24). «L'un des personnages les plus représentés par le cinéma», Jeanne d'Arc est abordée ici sous trois angles: les modèles formels à la base des films qui lui sont consacrés (narrations «en tableaux», fresques «épiques», chronique etc.), les épisodes évoqués par le nom même de la Pucelle et les choix opérés par les films, les visages enfin des actrices qui l'ont incarnée (chapitre 25). Parmi d'autres «libérateurs nationaux», Guillaume Tell a joui d'une certaine fortune cinématographique (chapitre 26). Pour ce qui est du dernier siècle du Moyen Âge, l'époque de Louis XI, avec le conflit franco-bourguignon et les troubles sociaux de la fin $\mathrm{du} \mathrm{Xv}^{\mathrm{e}}$ siècle, apparaît filtrée par Walter Scott d'une part, Victor Hugo de l'autre (chapitre 27).

6 Une section ultérieure renvoie au Fantasme moyenâgeux (pp. 905-998), à savoir ce que le cinéma à pu récupérer d'un héritage culturel riche et en partie seulement étranger. Récits de miracles (surtout miracles de la Vierge) et légendes profanes, bien que difficiles à séparer, ont souvent joué une «fonction compensatoire» dans la mesure où ils permettent de rapporter au Moyen Âge des conflits qui persistent de nos jours (chapitre 28). Les inquiétudes des temps modernes trouvent dans le cinéma la possibilité de se projeter dans un Ailleurs et un Autrefois, en créant un répertoire fantastique qui comprend des décors ou des sujets devenus typiques, parfois de convention: diables, loups-garous, dragons, êtres surnaturels peuplent des milieux considérés comme «médiévaux» dans l'imaginaire courant, sans que le sujet des films soit proprement situé au Moyen Âge (chapitre 29). Il existe enfin une catégorie nommée ici «Moyen Âge trivial» (p. 979), qui permet de regrouper les films exploitant les tonalités érotique et grivoise, souvent dans un cadre comique; le corpus est dans ce cas hétérogène, comprenant tant les films proposant une image contraire aux récits courtois que ceux qui se situent plutôt dans la veine des fabliaux et des farces, sources du rire «médiéval» (chapitre 30).

7 Pour terminer, on revient à des questions de portée générale, permettant de tirer quelques conclusions (cinquième partie, Comment représenter le Moyen Âge?, pp. 1001-1087). Il s'agit premièrement de faire la différence entre l'image médiévale et celle que propose le cinéma: cette irréductibilité n'est évidemment pas sans conséquence dans la représentation filmique, quelle que soit la solution - plus ou moins «archéologique» - adoptée; un problème analogue se pose pour les couleurs et le rendu 
chromatique (chapitre 31). Un essai de synthèse imposerait de trouver des «unités de contenu» minimales, identifiables dans quelques grands thèmes narratifs (trahison, usurpation, conflits familiaux) et concepts-cadres (chevalerie, féodalité, pouvoir temporel vs pouvoir spirituel); des topoi enfin, de longueur variable, se reconnaissent: bal, banquet, bataille, combat individuel, bûcher, cérémonies diverses, interventions de jongleurs et autres acteurs, siège, tournoi, joute, constituent autant de scènes récurrentes (chapitre 32) qui contribuent, avec les motifs les plus petits (armure, crénelage, croix, échecs, épée et arc, ogive et voûte, pignon, flèche, hennin) à reconstituer le monde médiéval au cinéma (chapitre 33).

8 Au total, ce gros ouvrage de F. Amy de la Brétèque aura répondu aux deux questions fondamentales qu'il a posées: «Que reste-t-il de l'imaginaire médiéval dans le cinéma? Et quelles représentations imaginaires du Moyen Âge le cinéma [...] a-t-il construites?» (p. 1089). Cet imaginaire est «une construction, un répertoire constitué d'éléments hétérogènes» (p.1092) qui permettent à la fois de réinterpréter le passé des civilisations occidentales, de revisiter les restes médiévaux dans le monde présent, parfois de faire du Moyen Âge un univers de fantaisie. Dans un tel cadre, le point de vue des «médiévistes», historiens, littéraires, historiens de l'art, n'est pas essentiel, le cinéma ayant de toute manière contribué à représenter une époque crédible en évitant les voies de la «reconstitution».

Outre la liste des titres, les compléments comprennent une bibliographie et sitographie raisonnée (pp.1227-1236), l'Index des noms (pp.1237-1248), l'Index des titres (pp. 1249-1265), une précieuse Concordance des titres originaux des films et des titres français (pp. 1267-1271).

La valeur de cet ouvrage tient aussi à la double compétence d'un Auteur, à la fois littéraire et cinématographique, dont les deux passions ont indubitablement enrichi une réflexion qui n'a rien perdu de sa fraîcheur quinze ans après sa première publication. 